\title{
On Super Strong ETH
}

Nikhil Vyas

Ryan Williams

CSAIL, MIT

Cambridge, MA 02139 USA
NIKHILV@MIT.EDU

RRW@MIT.EDU

\begin{abstract}
Multiple known algorithmic paradigms (backtracking, local search and the polynomial method) only yield a $2^{n(1-1 / O(k))}$ time algorithm for k-SAT in the worst case. For this reason, it has been hypothesized that the worst-case k-SAT problem cannot be solved in $2^{n(1-f(k) / k)}$ time for any unbounded function $\mathrm{f}$. This hypothesis has been called the "Super-Strong ETH", modeled after the ETH and the Strong ETH.

It has also been hypothesized that k-SAT is hard to solve for randomly chosen instances near the "critical threshold", where the clause-to-variable ratio is such that randomly chosen instances are satisfiable with probability $1 / 2$. We give a randomized algorithm which refutes the Super-Strong ETH for the case of random k-SAT and planted k-SAT for any clause-to-variable ratio. For example, given any random k-SAT instance $\mathrm{F}$ with $\mathrm{n}$ variables and $\mathrm{m}$ clauses, our algorithm decides satisfiability for $\mathrm{F}$ in $2^{n(1-\Omega(\log k) / k)}$ time, with high probability (over the choice of the formula and the randomness of the algorithm). It turns out that a well-known algorithm from the literature on SAT algorithms does the job: the PPZ algorithm of Paturi, Pudlak, and Zane (1999).

The Unique k-SAT problem is the special case where there is at most one satisfying assignment. Improving prior reductions, we show that the Super-Strong ETHs for Unique $\mathrm{k}$-SAT and k-SAT are equivalent. More precisely, we show the time complexities of Unique k-SAT and k-SAT are very tightly correlated: if Unique k-SAT is in $2^{n(1-f(k) / k)}$ time for an unbounded $\mathrm{f}$, then $\mathrm{k}$-SAT is in $2^{n(1-f(k) /(2 k))}$ time.
\end{abstract}

\section{Introduction}

The canonical NP-complete problem is $k$-SAT, for $k \geq 3$ : Given a Boolean formula in conjunctive normal form with clauses of width at most $k$, is it satisfiable? In practice, $k$-SAT is often cited as a "solved problem" (Gomes, Kautz, Sabharwal, \& Selman, 2008), due to the incredible performance of modern SAT solvers on instances arising from practice (mostly hardware and software verification). However, it is very possible that in the future, the demands and designs from practice will change significantly, leading to significantly different SAT instances. In general, the worst-case complexity of $k$-SAT is far from understood, in spite of tremendous effort devoted to finding faster worst-case algorithms. Because it is widely believed that $\mathrm{P} \neq \mathrm{NP}$, the search has been confined to super-polynomial-time algorithms. Although is trivial to obtain an algorithm running in $2^{n} \cdot m^{O(1)}$ time on $k$ SAT instances with $m$ clauses and $n$ variables, we cannot seem to improve the base of the exponent below 2: there are are no known algorithms for $k$-SAT which run in $(2-\epsilon)^{n} \cdot m^{O(1)}$ time for a universal constant $\epsilon>0$, independent of $k$. This apparent barrier to algorithms led researchers to the following two popular hypotheses which strengthen $\mathrm{P} \neq \mathrm{NP}$ : 
- Exponential Time Hypothesis (ETH) (Impagliazzo \& Paturi, 2001) There is an $\alpha>0$ such that no 3-SAT algorithm runs in $2^{\alpha n}$ time.

- Strong Exponential Time Hypothesis (SETH) (Calabro, Impagliazzo, \& Paturi, 2009) There does not exist a constant $\epsilon>0$ such that for all $k, k$-SAT can be solved in $(2-\epsilon)^{n}$ time.

In fact, the present situation for worst-case $k$-SAT algorithms looks even worse than hypothesized. The current best known algorithms for $k$-SAT all have running time bounds of the form

$$
2^{n\left(1-\Omega\left(\frac{1}{k}\right)\right)} .
$$

In other words, all time bounds have the form $2^{n\left(1-\frac{c}{k}\right)}$ for some fixed constant $c>0$. It is a very interesting phenomenon that the same running time upper bound is achieved by radically different algorithmic paradigms, such as randomized backtracking (Paturi, Pudlák, \& Zane, 1999; Paturi, Pudlák, Saks, \& Zane, 2005), local search (Schöning, 1999), the polynomial method (Chan \& Williams, 2016), and linear programming based methods (Brakensiek \& Guruswami, 2019). Even for simpler variants such as Unique- $k$-SAT (where we are promised there is at most one satisfying assignment), no significantly faster algorithms are known with a better dependence on $k$ in the exponent. Hence it is possible that the runtime behavior of $2^{n\left(1-\Omega\left(\frac{1}{k}\right)\right)}$ is actually optimal for $k$-SAT algorithms. This was termed the "Super-Strong ETH" in a 2015 talk by the second author (Williams, 2015). We state the Super-SETH as follows:

\section{Super-SETH: Super Strong Exponential Time Hypothesis.}

For every unbounded function $f: \mathbb{N} \rightarrow \mathbb{N}$, there is no (randomized) algorithm for $k$-SAT running in $O\left(2^{n\left(1-\frac{f(k)}{k}\right)}\right)$ time.

Intuitively, Super-SETH says that the $\Omega(1 / k)$ "savings" in the exponent is optimal: not even an $f(k) / k$ savings can be achieved, for any unbounded $f$. In this paper, we study Super-SETH in two natural restricted scenarios:

- Random/Planted $k$-SAT. We consider two general cases: (a) finding solutions to random $k$-SAT instances where each of the $m$ clauses is drawn uniformly and independently from all possible $k$-width clauses, and (b) finding solutions to planted $k$-SAT instances, where a random (hidden) solution $\sigma$ is sampled, then each clause is drawn uniformly and independently from all possible $k$-width clauses that satisfy $\sigma$.

Random $k$-SAT has a well-known threshold behaviour in which, for $\alpha_{\text {sat }}=2^{k} \ln 2-$ $\Theta(1)$ and for all constant $\epsilon>0$, random $k$-SAT instances are SAT w.h.p. (with high probability) for $m<\left(\alpha_{s a t}-\epsilon\right) n$ and UNSAT w.h.p. for $m>\left(\alpha_{s a t}+\epsilon\right) n$. Note that, as far as decidability is concerned, for instances below (respectively, above) the threshold we may simply output "SAT" (respectively, "UNSAT") and we will be correct w.h.p.. It has been conjectured (Cook \& Mitchell, 1996; Selman, Mitchell, \& Levesque, 1996) that random instances at the threshold $m=\alpha_{\text {sat }} n$ are the hardest random instances, and it is difficult to determine their satisfiability. We are motivated by the following strengthening of this conjecture.

Are random instances near the threshold as hard as the worst-case instances of $k$-SAT? 
- Unique $k$-SAT. This is the special case of finding a SAT assignment to a $k$-CNF, when one is promised that there is at most one satisfying assignment. It is wellknown to be NP-complete under randomized reductions (Valiant \& Vazirani, 1986). As mentioned earlier, the best known algorithms for Unique- $k$-SAT have the same running time behaviour of $2^{n\left(1-O\left(\frac{1}{k}\right)\right)}$ as $k$-SAT. In fact some of the best-known $k$ SAT algorithms (Paturi et al., 1999, 2005) have an easier analysis when restricted to the case of Unique- $k$-SAT. PPSZ (Paturi et al., 2005), the current best known algorithm for $k$-SAT (when $k \geq 5$ ) has only been derandomized for Unique- $k$-SAT.

\section{Could worst-case algorithms for Unique $k$-SAT be marginally faster than those for $k$-SAT?}

In principle, in this "ultra fine-grained" setting we are studying (where the exponential dependence on $k$ matters), both above special cases could potentially be just as hard as $k$-SAT, or both of them could be easier. In this paper, we prove that Super-SETH is false for Random $k$-SAT, and the Super-SETH for Unique $k$-SAT is equivalent to the general Super-SETH: the dependence on $k$ in the exponent is the same for the two problems.

\subsection{Prior Work}

As mentioned earlier, many algorithmic paradigms have been introduced for solving $k$-SAT in the worst case, but none are known to run in $2^{n\left(1-\omega_{k}(1 / k)\right)}$ time. There also has been substantial work on polynomial-time algorithms for random $k$-SAT that return solutions for $m$ below the threshold. Note that even though we know that these instances are satisfiable w.h.p. that does not immediately give a way to find a solution. Chao and Franco (1990) first proved that the unit clause heuristic (the key component of the PPZ algorithm) finds solutions with high probability for random $k$-SAT when $m \leq c 2^{k} n / k$ for some constant $c>0$. The current best known polynomial-time algorithm in this regime is by CojaOghlan (2010) and it can find a solution w.h.p. for random $k$-SAT when $m \leq c 2^{k} n \log (k) / k$ for some constant $c>0$. Interestingly, we also know of polynomial time algorithms for large $m$. Specifically, it is known that for a certain constant $C_{0}=C(k)$ and $m>C_{0} \cdot n$ there are polynomial-time algorithms finding solutions to planted $k$-SAT instances by Krivelevich and Vilenchik (2006) and random $k$-SAT (conditioned on satisfiability) by Coja-Oghlan et al. (2007). However, both of these results require that $m \geq 4^{k} n / k$ (Vilenchik, 2019). To our knowledge, no improvements over worst-case $k$-SAT algorithms have yet been reported for random $k$-SAT very close to the threshold.

Valiant and Vazirani (1986) gave a poly-time randomized reduction from SAT instances $F$ on $n$ variables to Unique-SAT instances $F^{\prime}$ on $n$ variables such that, if $F$ is SAT then $F^{\prime}$ has a unique satisfying assignment with probability at least $\Omega(1 / n)$, and if $F$ is UNSAT then $F^{\prime}$ is UNSAT. This reduction is not directly applicable for us to convert $k$-SAT instances into Unique- $k$-SAT instances, as they do not preserve the clause width (and when we perform a reduction to reduce the clause width, the number of new variables increases too much for exponential-time algorithms). To address this issue, Calabro et al. (2008) gave a randomized polynomial-time reduction with one-sided error from $k$-SAT to Unique- $k$-SAT which works with probability $2^{-O\left(n \log ^{2}(k) / k\right)}$. The probability bound was further improved by Traxler $(2008)$ to $2^{-O(n \log (k) / k)}$. Both of these reductions imply that k-SAT and Unique 
k-SAT either both have $2^{\delta n}$ time algorithms for some universal $\delta<1$, or neither of them do: in other words, the SETH and the "SETH for Unique- $k$-SAT" are equivalent statements. However these results are not sufficient for an equivalence with respect to Super-SETH: for example, from these results it is still possible that $k$-SAT has no $2^{n\left(1-\omega_{k}(1 / k)\right)}$ time algorithms, while Unique- $k$-SAT has a $2^{n(1-\Omega(\log k / k))}$ time algorithm.

\subsection{Our Results}

In the next two subsections we present our main results on Random $k$-SAT and Unique $k$-SAT respectively.

\subsubsection{Average-Case $k$-SAT Algorithms}

First we present an algorithm which breaks the Super-Strong ETH for random $k$-SAT. In particular, we give a $2^{n\left(1-\Omega\left(\frac{\log k}{k}\right)\right)}$-time algorithm which finds a solution w.h.p. for randomk-SAT (conditioned on satisfiability) for all values of $m$. In fact, our algorithm is an old one from the SAT algorithms literature: the PPZ algorithm of Paturi et al. (Paturi et al., 1999).

In order to show that PPZ breaks Super-Strong ETH in the random case, we first show that PPZ yields a faster algorithm for random planted $k$-SAT for large enough $m$.

Theorem 1. There is a randomized algorithm that, given a planted $k$-SAT instance $F$ sampled from $P(n, k, m)]^{1}$ with $m>2^{k-1} \ln (2)$, outputs a satisfying assignment to $F$ in $2^{n\left(1-\Omega\left(\frac{\log k}{k}\right)\right)}$ time with $1-2^{-\Omega\left(n\left(\frac{\log k}{k}\right)\right)}$ probability (over the planted $k$-SAT distribution and the randomness of the algorithm).

The main idea behind Theorem 1 is to effectively estimate how often unit propagation can be applied to a random $k$-SAT instance. (Recall that "unit propagation" says that any clause containing only one literal can be simplified, by simply setting the literal to its appropriate value, reducing the number of variables by one.) In Lemma 6 we show that, when variables are set randomly and unit propagation is being applied as aggressively as possible, a surprisingly large number of variables $(\Omega(n \log k) / k)$ will get assigned by unit propagation, reducing the overall expected running time by a factor of $2^{\Omega(n \log k) / k}$.

Next, we give a reduction from random $k$-SAT (conditioned on satisfiability, we denote this distribution by $R^{+}$) to planted $k$-SAT. Similar reductions/equivalences have been observed before by Ben-Sasson et al. (2002) and Achlioptas and Coja-Oghlan (2008).

Theorem 2. Suppose there is an algorithm $A$ for planted $k$-SAT $P(n, k, m)$, for all $m \geq$ $2^{k} \ln 2(1-f(k) / 2) n$, which finds a solution in time $2^{n(1-f(k))}$ and with probability $1-2^{-n f(k)}$, where $1 / k<f(k)=o_{k}(1)$. Then for any $m^{\prime}$, given a random $k$-SAT instance sampled from $R^{+}\left(n, k, m^{\prime}\right)$, a satisfying assignment can be found in $2^{n(1-\Omega(f(k)))}$ time w.h.p.

Combining Theorems 1 and 2 yields:

Theorem 3. Given a random $k$-SAT instance $F$ sampled from $R^{+}(n, k, m)$, we can find a solution in $2^{n\left(1-\Omega\left(\frac{\log k}{k}\right)\right)}$ time w.h.p., for any $m$.

1. See "Three $k$-SAT Distributions" in Section 2 for formal definitions of different $k$-SAT distributions. 
Remark 1. There are other interesting and natural hypotheses for random $k$-SAT that we do not resolve. We obtain a randomized algorithm for random $k$-SAT which always reports UNSAT on unsatisfiable instances, and finds a SAT assignment with high probability on satisfiable instances. Feige's Hypothesis for $k$-SAT (Feige, 2002) conjectures that there are no efficient refutations for random $k$-SAT near the threshold, i.e., there are no efficient algorithms which always report SAT on satisfiable instances, and report UNSAT on unsatisfiable instances with probability at least 1/2. Refuting Feige's hypothesis in our setting remains an intriguing open problem.

Theorems 1 and 3 imply that at least one of the following are true:

1. Either the random instances of $k$-SAT at the threshold are not the hardest instances of $k$-SAT, or

2. Super-Strong ETH is false.

For the PPZ algorithm (randomized branching with unit propagation) and its generalization PPSZ (Paturi et al., 2005), time lower bounds of the form $2^{n\left(1-O\left(\frac{1}{k}\right)\right)}$ are in fact known (Pudlák, Scheder, \& Talebanfard, 2017; Scheder \& Talebanfard, 2020). Thus we can say that, with respect to the PPZ/PPSZ algorithm, random $k$-SAT instances are provably more tractable than worst-case $k$-SAT instances.

In Section 5, we observe that our techniques can be used to get algorithms running faster than $2^{n\left(1-\Omega\left(\frac{\log k}{k}\right)\right)}$ for planted $k$-SAT and random $k$-SAT (conditioned on satisfiability), depending on how large $m / n$ is compared to the threshold density.

\subsubsection{UNIQUE $k$-SAT EQUIVALENCE}

In Section 6 we give a "low exponential" time reduction from $k$-SAT to Unique- $k$-SAT, which proves that the two problems are equivalent with respect to Strong-SETH. More precisely, we show that there is a $2^{n\left(1-\omega_{k}(1 / k)\right)}$ time algorithm for Unique- $k$-SAT if and only if there is a $2^{n\left(1-\omega_{k}(1 / k)\right)}$ time algorithm for $k$-SAT. In fact, our reduction has the following stronger property:

Theorem 4. If Unique $k$-SAT is solvable in $2^{(1-f(k) / k) n}$ time for some unbounded function $f$, then $k$-SAT is solvable in $2^{(1-f(k) / k+O(\log (f(k)) / k)) n}$ time.

As mentioned earlier, the current best algorithm for $k$-SAT PPSZ (Paturi et al., 2005) has a much easier analysis for Unique $k$-SAT, and in fact it was an open question to show that its running time on general instances of $k$-SAT matches the running time for Unique $k$-SAT; this was eventually resolved by Hertli (2014). Theorem 4 implies that, in order to obtain faster algorithms for $k$-SAT which break Super-Strong ETH, it would be sufficient to restrict ourselves to Unique $k$-SAT, which might simplify the analysis as in the case of PPSZ.

\section{Preliminaries}

Notation. In this paper, we generally assume $k \geq 3$ is an arbitrarily large integer. Throughout the paper, we compare time bounds that have the form $2^{n(1-\Omega(\log k) / k)}$ with 
$2^{n(1-O(1 / k))}$ time, where the big- $\Omega$ and the big- $O$ hide multiplicative constants which are independent of both $n$ and $k$; such notation only makes sense when $k$ can grow unboundedly. We will assume throughout the paper that for a formula $F$ over $n$ variables the number of clauses $m$ is less than $\operatorname{poly}(n)$.

We use the terms "solution", "SAT assignment", and "satisfying assignment" interchangeably. For an $n$-variable assignment $s \in\{0,1\}^{n}$ and an index set $I \subseteq[n]$, we use $s_{\mid I}$ to denote the length- $|I|$ substring of $s$ projected on the index set $I$. We use the notation $x \in_{r} \chi$ to denote that $x$ is randomly sampled from the distribution $\chi$. By $\operatorname{poly}(n)$, we mean some function $f(n)$ which satisfies $f(n)=O\left(n^{c}\right)$ for a universal constant $c \geq 1$. Letting $n$ be the number of variables in a $k$-CNF, a random event about $k$-CNF holds w.h.p. (with high probability) if it holds with probability $1-f(n)$, where $f(n) \rightarrow 0$ as $n \rightarrow \infty$. We use log and ln to denote the logarithm base- 2 and base- $e$ respectively, and $H(p)=-p \log (p)-(1-p) \log (1-p)$ denotes the binary entropy function. For a function $f(x)$ we denote its derivative and double-derivative by $f^{\prime}(x)$ and $f^{\prime \prime}(x)$ respectively.

Three $k$-SAT Distributions. We consider three distributions for random $k$-SAT:

- $R(n, k, m)$ is the distribution over formulas $F$ of $m$ clauses over $n$ variables, where each clause is drawn i.i.d. from the set of all $k$-width clauses. This is the standard $k$-SAT distribution.

- $R^{+}(n, k, m)$ is the distribution over formulas $F$ of $m$ clauses over $n$ variables where each clause is drawn i.i.d. from the set of all $k$-width clauses and we condition $F$ on being satisfiable i.e. $R(n, k, m)$ conditioned on satisfiability.

- $P(n, k, m, \sigma)$ is the distribution over formulas $F$ of $m$ clauses over $n$ variables where each clause is drawn i.i.d. from the set of all $k$-width clauses which satisfy $\sigma . P(n, k, m)$ is the distribution over formulas $F$ formed by sampling $\sigma \in\{0,1\}^{n}$ uniformly and then sampling $F$ from $P(n, k, m, \sigma)$.

Note that an algorithm solving the search problem (finding SAT assignments) for instances sampled from $R^{+}$is stronger than deciding satisfiability for instances sampled from $R$ : given an algorithm for the search problem on $R^{+}$, we may run it on a random instance from $R$, returning SAT if and only if the algorithm returns a valid satisfying assignment.

\subsection{Structural Properties of Planted and Random k-SAT}

A few structural results about planted and random $k$-SAT will be useful in analyzing our algorithms. In particular, we consider bounds on the expected number of solutions of planted $k$-SAT instances and random $k$-SAT instances (conditioned on satisfiability).

A well-known conjecture is that the satisfiability of random $k$-SAT displays a threshold behaviour for all $k$. The following lemma which states that the conjecture holds for all $k$ (larger than a fixed constant) was proven by Ding et al. (2015).

Lemma 1 (Ding et al., 2015). There is a constant $k_{0}$ such that for all $k>k_{0}$, there exists an $\alpha_{\text {sat }}=2^{k} \ln 2-\Theta(1)$ and for all constant $\epsilon>0$, we have that:

$$
\begin{aligned}
& \text { For } m<(1-\epsilon) \alpha_{\text {sat }} n, \lim _{n \rightarrow \infty} \operatorname{Pr}_{F \in r} \operatorname{Pr}_{(n, k, m)}[F \text { is satisfiable }]=1 \\
& \text { For } m>(1+\epsilon) \alpha_{\text {sat }} n, \lim _{n \rightarrow \infty} \operatorname{Pr}_{F \in r} \operatorname{Pr}_{(n, k, m)}[F \text { is satisfiable }]=0
\end{aligned}
$$


We will also need the fact that, w.h.p., the ratio of the number of solutions and its expected value is not too small, as long as $m$ is a bit below the satisfiability threshold. Quantitatively, we will take $m \leq \alpha_{d} n$ where $\alpha_{d}=2^{k} \ln 2-k$ (note that $\alpha_{d}<\alpha_{\text {sat }}-1$ ).

Lemma 2 (Lemma 22 of Achlioptas \& Coja-Oghlan, 2008). For $F \in_{r} R(n, k, m)$, let $\mathcal{S}$ be the set of solutions of $F$. Then $E[|\mathcal{S}|]=2^{n}\left(1-\frac{1}{2^{k}}\right)^{m}$. Furthermore, for $\alpha_{d}=2^{k} \ln 2-k$ and $m \leq \alpha_{d} n$ we have

$$
\lim _{n \rightarrow \infty} \operatorname{Pr}\left[|\mathcal{S}|<E[|\mathcal{S}|] / 2^{O\left(n k / 2^{k}\right)}\right]=0
$$

Together, the above two results have the following useful consequence. Intuitively, the below lemma states that if our random $k$-SAT instance is slightly below the threshold, then (conditioned on being satisfiable) we can fairly tightly bound the expected number of SAT assignments.

Lemma 3. For $F \in_{r} R^{+}(n, k, m)$ let $Z$ denote the number of solutions of $F$. For all constant $\delta>0$, if $m<(1-\epsilon) \alpha_{\text {sat }} n$ for some constant $\epsilon>0$, then for all large enough $n$,

$$
2^{n}\left(1-\frac{1}{2^{k}}\right)^{m} \leq E[Z] \leq(1+\delta) 2^{n}\left(1-\frac{1}{2^{k}}\right)^{m}
$$

. Furthermore, for $\alpha_{d}=2^{k} \ln 2-k$ and $m \leq \alpha_{d} n$,

$$
\lim _{n \rightarrow \infty} \operatorname{Pr}\left[Z<E[Z] / 2^{O\left(n k / 2^{k}\right)}\right]=0 .
$$

Proof. Let $F^{\prime} \in_{r} R(n, k, m)$ and let $Z^{\prime}$ denote the number of solutions of $F^{\prime}$. By Lemma 2, $E\left[Z^{\prime}\right]=2^{n}\left(1-\frac{1}{2^{k}}\right)^{m}$. Letting $p_{n}$ denote the probability that $F^{\prime}$ is unsatisfiable, we have $E\left[Z^{\prime}\right]=\left(1-p_{n}\right) E[Z]$. As $m<(1-\epsilon) \alpha_{\text {sat }}$, by Lemma 1 $\lim _{n \rightarrow \infty} p_{n} \rightarrow 0$, hence $2^{n}\left(1-\frac{1}{2^{k}}\right)^{m} \leq$ $E[Z] \leq(1+\delta) 2^{n}\left(1-\frac{1}{2^{k}}\right)^{m}$ for all constants $\delta$.

Observe that $\operatorname{Pr}\left[Z<E[Z] / 2^{O\left(n k / 2^{k}\right)}\right] \leq \operatorname{Pr}\left[Z^{\prime}<E[Z] / 2^{O\left(n k / 2^{k}\right)}\right]$, as $Z$ is just $Z^{\prime}$ conditioned on being positive. Furthermore $\operatorname{Pr}\left[Z^{\prime}<E[Z] / 2^{O\left(n k / 2^{k}\right)}\right] \leq \operatorname{Pr}\left[Z^{\prime}<E\left[Z^{\prime}\right] / 2^{O\left(n k / 2^{k}\right)}\right]$ as $E[Z] \leq 2 E\left[Z^{\prime}\right]$ from the previous paragraph. By Lemma 2, $\lim _{n \rightarrow \infty} \operatorname{Pr}\left[Z^{\prime}<E\left[Z^{\prime}\right] / 2^{O\left(n k / 2^{k}\right)}\right]=$ 0 .

We will use a planted k-SAT algorithm to solve random k-SAT instances conditioned on their satisfiability. This idea was introduced in an unpublished manuscript by Ben-Sasson et al. (2002). We will use the following lemma therein.

Lemma 4 (Lemma 3.3 of Ben-Sasson et al., 2002). For a given $F$ in $R^{+}(n, k, m)$, let $Z$ denote its number of solutions. $F$ is sampled from $P(n, k, m)$ with probability $Z \cdot p$, where $p$ only depends on $n, k$, and $m$.

Proof. For a fixed $\sigma, P(n, k, m, \sigma)$ is just the uniform distribution over all $k$-SAT formulas $F$ with $m$ clauses over $n$ variables which satisfy $\sigma$. Let there be $t$ of such formulas then each one is sampled with probability $1 / t$. Note that $t$ depends only on $n, k, m$ and not on $\sigma$.

Let $F$ be a $k$-SAT formula with $m$ clauses over $n$ variables which has $Z$ solutions. Then by the definition of $P(n, k, m)$ probability that a sample from $P(n, k, m)$ equals $F$ is $Z /\left(t 2^{n}\right)$. Setting $p=1 /\left(t 2^{n}\right)$ completes the proof. 
Corollary 1. For $F \in_{r} R^{+}(n, k, m)$ and $F^{\prime} \in_{r} P(n, k, m)$ let $Z$ and $Z^{\prime}$ denote their number of solutions respectively. Then for $\alpha_{d}=2^{k} \ln 2-k$ and for $m \leq \alpha_{d} n, \lim _{n \rightarrow \infty} \operatorname{Pr}\left[Z^{\prime}<\right.$ $\left.E[Z] / 2^{O\left(n k / 2^{k}\right)}\right]=0$.

Proof. We have $\lim _{n \rightarrow \infty} \operatorname{Pr}\left[Z<E[Z] / 2^{O\left(n k / 2^{k}\right)}\right]=0$ by Lemma 3 . Lemma 4 shows that the planted $k$-SAT distribution $P(n, k, m)$ is biased toward satisfiable formulas with more solutions. The distribution $R^{+}(n, k, m)$ instead chooses all satisfiable formulas with equal probability. Hence $\lim _{n \rightarrow \infty} \operatorname{Pr}\left[Z^{\prime}<E[Z] / 2^{O\left(n k / 2^{k}\right)}\right] \leq \lim _{n \rightarrow \infty} \operatorname{Pr}\left[Z<E[Z] / 2^{O\left(n k / 2^{k}\right)}\right]=$ 0 .

So far, our lemmas have only been applicable where $m \leq \alpha_{d} n \leq\left(\alpha_{\text {sat }}-1\right) n$. Next we prove a lemma bounding the number of expected solutions when $m \geq\left(\alpha_{\text {sat }}-1\right) n$.

Lemma 5. For $m \geq\left(\alpha_{\text {sat }}-1\right) n$, the expected number of solutions of $F \in_{r} R^{+}(n, k, m)$ and $F^{\prime} \in_{r} P(n, k, m)$ is at most $2^{O\left(n / 2^{k}\right)}$ in both cases.

Proof. Lemma 4 shows that the planted $k$-SAT distribution $P(n, k, m)$ is biased toward satisfiable formulas with more solutions. In particular, the expected number of solutions of $F^{\prime} \in_{r} P(n, k, m)$ upper bounds the expected number for $F \in_{r} R^{+}(n, k, m)$. So it suffices to upper bound the expected number of solutions of $F^{\prime} \in_{r} P(n, k, m)$.

Let $Z$ be the random variable denoting the number of solutions of $F^{\prime}$. Let $\sigma$ denote the planted solution in $F$, and let $x$ be some assignment which has hamming distance $i$ from $\sigma$. For a clause $C$ satisfied by $\sigma$ but not by $x$, all of $C$ 's satisfied literals must come from the $i$ bits where $\sigma$ and $x$ differ, and all its unsatisfied literals must come from the remaining $n-i$ bits. Letting $j$ denote the number of satisfying literals in $C$, the probability that a randomly sampled clause $C$ is satisfied by $\sigma$ but not by $x$ is $\sum_{j=1}^{k} \frac{\left(\begin{array}{l}k \\ j\end{array}\right)}{2^{k}-1}\left(\frac{i}{n}\right)^{j}\left(1-\frac{i}{n}\right)^{k-j}=\frac{1-\left(1-\frac{i}{n}\right)^{k}}{2^{k}-1}$. 
We will now upper bound $E[Z]$.

$$
\begin{aligned}
E[Z] & =\sum_{y \in\{0,1\}^{n}} \operatorname{Pr}\left[y \text { satisfies } F^{\prime}\right] \\
& =\sum_{i=1}^{n}\left(\begin{array}{c}
n \\
i
\end{array}\right) \operatorname{Pr}\left[\text { Assignment } x \text { that differs from } \sigma \text { in } i \text { bits satisfies } F^{\prime}\right] \\
& =\sum_{i=1}^{n}\left(\begin{array}{c}
n \\
i
\end{array}\right) \operatorname{Pr}[\text { A random clause satisfying } \sigma \text { satisfies } x]^{m} \\
& =\sum_{i=1}^{n}\left(\begin{array}{c}
n \\
i
\end{array}\right)(1-\operatorname{Pr}[\text { A random clause satisfying } \sigma \text { does not satisfy } x])^{m} \\
& =\sum_{i=1}^{n}\left(\begin{array}{c}
n \\
i
\end{array}\right)\left(1-\frac{1-(1-i / n)^{k}}{2^{k}-1}\right)^{m} \quad[\text { As shown above }] \\
& \leq \sum_{i=1}^{n}\left(\begin{array}{c}
n \\
i
\end{array}\right) e^{-m\left(\frac{1-(1-i / n)^{k}}{2^{k}-1}\right)}\left[\text { As } 1-x \leq e^{-x}\right] \\
& \leq \sum_{i=1}^{n}\left(\begin{array}{c}
n \\
i
\end{array}\right) e^{-\left(\alpha_{s a t}-1\right) n\left(\frac{1-(1-i / n)^{k}}{2^{k}-1}\right)} \\
& \leq 2^{O\left(n / 2^{k}\right)} \sum_{i=1}^{n}\left(\begin{array}{c}
n \\
i
\end{array}\right) e^{-\left(\left(2^{k}-1\right) \ln 2\right) n\left(\frac{1-(1-i / n)^{k}}{2^{k}-1}\right)} \quad\left[\text { As } m \geq\left(2^{k} \ln 2-O(1)\right) n\right] \\
& \leq 2^{O\left(n / 2^{k}\right)} \sum_{i=1}^{n}\left(\begin{array}{c}
n \\
i
\end{array}\right) 2^{-n\left(1-(1-i / n)^{k}\right)} \\
& 2_{i=1}^{n} 2^{n\left(H(i / n)-1+(1-i / n)^{k}\right)} \leq 2^{O\left(n / 2^{k}\right)} \max 2^{n\left(H(p)-1+(1-p)^{k}\right)} .
\end{aligned}
$$

Let $f(p)=H(p)-1+(1-p)^{k}$. Then $f^{\prime}(p)=-\log \left(\frac{p}{1-p}\right)-k(1-p)^{k-1}$ and $f^{\prime \prime}(p)=$ $\frac{-1}{p(1-p)}+k(k-1)(1-p)^{k-2}$. Thus $f^{\prime \prime}(p)=0 \Longleftrightarrow p(1-p)^{k-1}=\frac{1}{k(k-1)}$. Note that $f^{\prime \prime}(p)$ has only two roots in $[0,1]$, hence $f^{\prime}(p)$ has at most 3 roots in $[0,1]$. It can be verified that for sufficiently large $k, f^{\prime}(p)$ indeed has three roots at $p=\Theta\left(1 / 2^{k}\right), \Theta(\log k / k)$, and $1 / 2-\Theta\left(k / 2^{k}\right)$. At all these three values of $p, f(p)=O\left(1 / 2^{k}\right)$. Hence $E[Z] \leq 2^{O\left(n / 2^{k}\right)}$.

\section{Planted k-SAT and the PPZ Algorithm}

In this section, we establish that the PPZ algorithm solves random planted $k$-SAT instances faster than $2^{n-n / O(k)}$ time.

Reminder of Theorem 1. There is a randomized algorithm that given a planted $k-S A T$ instance $F$ sampled from $P(n, k, m)$ with $m>2^{k-1} \ln (2)$, outputs a satisfying assignment to F in $2^{n\left(1-\Omega\left(\frac{\log k}{k}\right)\right)}$ time with $1-2^{-\Omega\left(n\left(\frac{\log k}{k}\right)\right)}$ probability (over the planted $k$-SAT distribution and the randomness of the algorithm).

We will actually prove a stronger claim: 
For any $\sigma$ and $F$ sampled from $P(n, k, m, \sigma)$, we can find a set $S$ of $2^{n\left(1-\Omega\left(\frac{\log k}{k}\right)\right)}$ variable assignments in $2^{n\left(1-\Omega\left(\frac{\log k}{k}\right)\right)}$ time, such that $\sigma \in S$ with probability $1-2^{-\Omega\left(n\left(\frac{\log k}{k}\right)\right)}$ (the probability is over the planted $k$-SAT distribution and the randomness of the algorithm).

Theorem 1 yields an algorithm that (always) finds a solution for $k$-SAT instance $F$ sampled from $P(n, k, m)$, and runs in expected time $2^{n\left(1-\Omega\left(\frac{\log k}{k}\right)\right)}$. In fact, the algorithm of Theorem 1 is a simplification of the PPZ algorithm (Paturi et al., 1999), a well-known worst case algorithm for $k$-SAT. PPZ runs in polynomial time, and outputs a SAT assignment (on any satisfiable $k$-CNF) with probability $p \geq 2^{-n+n / O(k)}$. It can be repeatedly run for $O(n / p)$ times to obtain a worst-case algorithm that is correct whp. We consider a simplified version which is sufficient for analyzing planted $k$-SAT:

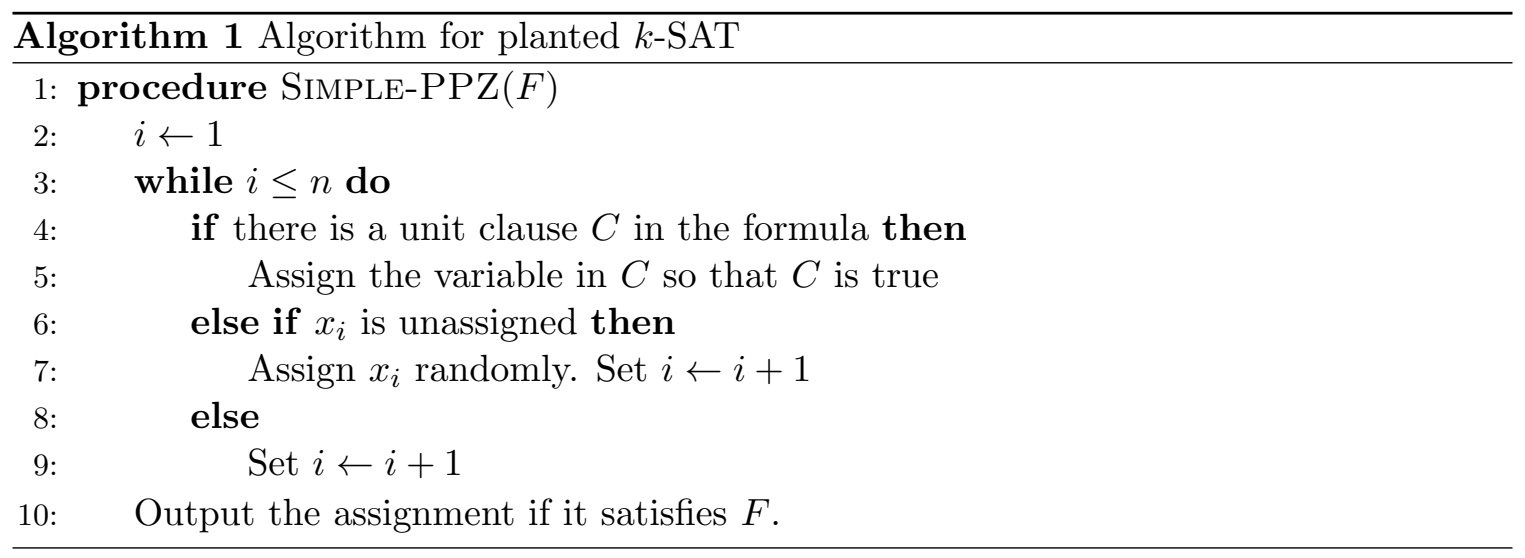

Our Simple-PPZ algorithm (Algorithm 1) only differs from PPZ in that PPZ also performs an initial random permutation of variables. For us, a random permutation is unnecessary: a random permutation of the variables in the planted $k$-SAT distribution yields the same distribution of instances. That is, the original PPZ algorithm would have the same behavior as Simple-PPZ over the distribution of instances from planted $k$-SAT.

Let the set of variables be $x_{1}, x_{2}, \ldots, x_{n}$. We will start with a few useful definitions.

Definition 1 (Paturi et al., 1999). A clause $C$ is critical with respect to variable $x_{i}$ and SAT assignment $\sigma$ if $x_{i}$ is the only variable in $C$ whose corresponding literal is satisfied by $\sigma$.

Definition 2. A variable $x_{i}$ in $F$ is good for an assignment $\sigma$ if there is a clause $C$ in $F$ which is critical with respect to $x_{i}$ and $\sigma$, and $i$ is the largest index among all variables in $C$. We say that $x_{i}$ is good with respect to $C$ in such a case. A variable which is not good is called bad.

Observe that for every good variable $x_{i}$, if all variables $x_{j}$ for $j<i$ are assigned correctly with respect to $\sigma$, then Simple-PPZ sets $x_{i}$ correctly, due to the unit clause rule. As such, given a formula $F$ with $z$ good variables for $\sigma$, the probability that Simple-PPZ finds $\sigma$ is at least $2^{-(n-z)}$ : if all $n-z$ bad variables are correctly assigned, the algorithm is forced to set all good variables correctly as well. Next, we prove a high-probability lower bound on the number of good variables in a random planted $k$-SAT instance. 
Lemma 6. For $m>n 2^{k-1} \ln 2$, a planted $k$-SAT instance sampled from $P(n, k, m, \sigma)$ has $\Omega(n \log k / k)$ good variables with respect to $\sigma$, with probability $1-2^{-\Omega\left(\frac{n \log k}{k}\right)}$.

Proof. Let $F \in_{r} P(n, k, m, \sigma)$ and let $L$ be the last (when sorted by index) $n \ln k /(2 k)$ variables. Let $L_{g}, L_{b}$ be the good and bad variables respectively, with respect to $\sigma$, among the variables in $L$. Let $E$ be the event that $\left|L_{g}\right| \leq n \ln k /(500 k)$. We will prove a strong upper bound on the probability that $E$ occurs. For all $x_{i} \in L$, we have that $i \geq n(1-$ $\ln k /(2 k))$. Observe that if a clause $C$ is such that $x_{i} \in L_{b}$ is good for $\sigma$ with respect to $C$, then $C$ does not occur in $F$. We will lower bound the probability of such a clause occurring in $F$, with respect to a fixed variable $x_{i} \in L$. Recall that in planted $k$-SAT, each clause is drawn uniformly at random from the set of clauses satisfied by $\sigma$. Fixing $\sigma$ and a variable $x_{i}$ and sampling one clause $C$, we get that

$$
\begin{aligned}
& C \text { which satisfies } \sigma \\
& =\frac{\operatorname{Pr}}{\text { total number of clauses satisfying } \sigma}=\frac{\left.x_{i} \in \text { is good with respect to } C\right]}{\left(\begin{array}{l}
n \\
k
\end{array}\right)\left(2^{k}-1\right)} \\
& \geq \frac{1}{2}\left(\frac{i}{n}\right)^{k-1} \frac{k}{2^{k} n} \quad[\text { As } i \geq n(1-\ln k /(2 k))] \\
& \geq \frac{1}{2}\left(\frac{i}{n}\right)^{k} \frac{k}{2^{k} n} \geq \frac{1}{2}\left(1-\frac{\ln k}{2 k}\right)^{k} \frac{k}{2^{k} n} \quad[\text { As } i \geq n(1-\ln k /(2 k))] \\
& \geq \frac{1}{2}\left(e^{-\ln k / k}\right)^{k} \frac{k}{2^{k} n} \quad\left[\text { As } k \text { is large, and } e^{-w} \leq 1-w / 2 \text { for small enough } w>0\right] \\
& \geq \frac{1}{2^{k+1} n}
\end{aligned}
$$

If the event $E$ is true, then $\left|L_{b}\right|=|L|-\left|L_{g}\right|>n \ln k /(4 k)$. Consider such a fixed set $L_{b}$. Under our sampling procedure, every time we sample a clause $C$, the probability that $C$ makes some variable $x_{i} \in L_{b} \operatorname{good}$ is at least $\frac{n \ln k}{4 k} \cdot \frac{1}{2^{k+1} n} \geq \frac{\ln k}{k 2^{k+3}}$, as the sets of clauses which make different variables good are disjoint sets. Now we upper bound the probability of $E$ occurring:

$$
\begin{aligned}
\operatorname{Pr}[E] & \leq \sum_{i=1}^{n \ln k /(500 k)} \operatorname{Pr}[\text { exactly } i \text { vars among the last } n \ln k /(2 k) \text { vars are good }] \\
& \leq \sum_{i=1}^{n \ln k /(500 k)}\left(\begin{array}{c}
n \ln k /(2 k) \\
i
\end{array}\right)\left(1-\frac{\ln k}{k 2^{k+3}}\right)^{m} \\
& \leq n\left(\begin{array}{c}
n \ln k /(2 k) \\
n \ln k /(500 k)
\end{array}\right)\left(1-\frac{\ln k}{k 2^{k+3}}\right)^{n 2^{k-1} \ln 2} . \quad\left[\text { As } m>n 2^{k-1} \ln 2\right]
\end{aligned}
$$

Applying the inequality $1-x \leq e^{-x}$ for $x>0$, the above is at most

$$
n\left(\begin{array}{c}
n \ln k /(2 k) \\
n \ln k /(500 k)
\end{array}\right)\left(e^{-\frac{\ln k}{k 2^{k+3}}}\right)^{n 2^{k-1} \ln 2} \leq n\left(\begin{array}{c}
n \ln k /(2 k) \\
n \ln k /(500 k)
\end{array}\right)\left(2^{-\frac{n \ln k}{16 k}}\right) \leq 2^{-\delta \frac{n \ln k}{k}}
$$

for appropriately small but constant $\delta>0$, which proves the lemma statement. 
We are now ready to prove Theorem 1 .

Proof of Theorem 1. By Lemma 6, we know that with probability at least $1-p$ for $p=$ $2^{-\Omega\left(n\left(\frac{\log k}{k}\right)\right)}$, the number of good variables with respect to a hidden planted solution $\sigma$ in $F$ is at least $\gamma n \log k / k$ for a constant $\gamma>0$. For such instances, a single run of PPZ outputs $\sigma$ with probability at least $2^{-n(1-\gamma \log k / k)}$. Repeating PPZ for $\operatorname{poly}(n) 2^{n(1-\gamma \log k / k)}$ times implies a success probability at least $1-1 / 2^{n}$. Hence the overall error probability is at most $p+1 / 2^{n} \leq 2^{-\Omega\left(n\left(\frac{\log k}{k}\right)\right)}$.

We proved that PPZ runs in time $2^{n\left(1-\Omega\left(\frac{\log k}{k}\right)\right)}$ when $m$ is "large enough", i.e., $m>$ $n 2^{k-1} \ln 2$. When $m \leq n 2^{k-1} \ln 2$, we observe that the much simpler approach of merely randomly sampling assignments already works, with high probability! This is because by Corollary 1 (in the Preliminaries), the number of solutions of $F \in_{r} P(n, k, m)$ for $m \leq n 2^{k-1} \ln 2$ is at least $2^{n / 2} 2^{-O\left(n k / 2^{k}\right)}$ with high probability. When this event happens, randomly sampling poly(n) $2^{n / 2} 2^{O\left(n k / 2^{k}\right)}$ assignments will uncover a solution with high probability.

\section{Reducing from Random $k$-SAT to Planted Random $k$-SAT}

In this section we observe a reduction from random k-SAT to planted k-SAT, which yields the desired algorithm for random k-SAT (see Theorem 3). The following lemma gives a reduction which preserves the number of clauses and is similar to results in Achlioptas (Achlioptas \& Coja-Oghlan, 2008), and we present it here for completeness.

Lemma 7 ((Achlioptas \& Coja-Oghlan, 2008)). Suppose there exists an algorithm A for planted $k$-SAT $P(n, k, m)$, for some $m \geq 2^{k} \ln 2(1-f(k) / 2) n$, which finds a solution in time $2^{n(1-f(k))}$ and with probability greater than $1-2^{-n f(k)}$, where $1 / k<f(k)=o_{k}(1) \mathrm{I}^{2}$ Then given a random $k$-SAT instance sampled from $R^{+}(n, k, m)$, we can find a satisfiable solution in $2^{n(1-\Omega(f(k)))}$ time with $1-2^{-n \Omega(f(k))}$ probability.

Proof. Let $F$ be sampled from $R^{+}(n, k, m)$, and let $Z$ denote its number of solutions with $s$ its expected value. As $f(k)>1 / k$ and $m \geq 2^{k} \ln 2(1-f(k) / 2) n$, Lemma 3 and 5 together imply that $s \leq 2 \cdot 2^{n f(k) / 2}$.

We will now run Algorithm $A$. Note that if Algorithm $A$ gives a solution it is correct hence we can only have error when the formula is satisfiable but algorithm $A$ does not return a solution. We will now upper bound the probability of $A$ making an error.

$$
\begin{aligned}
& \underset{F \in R^{+}(n, k, m), A}{\operatorname{Pr}}[A \text { returns no solution }] \\
\leq & \sum_{\sigma \in\{0,1\}^{n}} \operatorname{Pr}_{F \in R^{+}(n, k, m), A}[\sigma \text { satisfies } F \text { but } A \text { returns no solution }] \\
\leq & \sum_{\sigma \in\{0,1\}^{n}} \operatorname{Pr}_{F \in R^{+}(n, k, m), A}[A \text { returns no sol } \mid \sigma \text { satisfies } \mathrm{F}] \underset{F \in R^{+}(n, k, m)}{\operatorname{Pr}}[\sigma \text { satisfies } \mathrm{F}] \\
\leq & \sum_{\sigma \in\{0,1\}^{n}} \operatorname{Pr}_{F \in P(n, k, m, \sigma), A}[A \text { returns no solution }] \underset{F \in R^{+}(n, k, m)}{\operatorname{Pr}}[\sigma \text { satisfies } \mathrm{F}]
\end{aligned}
$$

2. Note we can assume wlog that $f(k)>1 / k$, as we already have a $2^{n(1-1 / k)}$ algorithm for worst-case $k$-SAT. 
where the last inequality used the fact that $R^{+}(n, k, m)$ conditioned on having $\sigma$ as a solution is the distribution $P(n, k, m, \sigma)$. Now note that $\operatorname{Pr}_{F \in R^{+}(n, k, m)}[\sigma$ satisfies $\mathrm{F}]=s / 2^{n}$, and $P(n, k, m)=P(n, k, m, \sigma)$, where $\sigma$ is sampled uniformly from $\{0,1\}^{n}$. Hence the expression simplifies to

$$
\begin{aligned}
& \frac{s}{2^{n}}\left(2^{n} \underset{F \in P(n, k, m), A}{\operatorname{Pr}}[A \text { does not return a solution }]\right) \\
& =s \operatorname{Pr}_{F \in P(n, k, m), A}[A \text { does not return a solution }] .
\end{aligned}
$$

Since $s \leq 2 \cdot 2^{n f(k) / 2}$, the error probability is $\leq 2 \cdot 2^{n f(k) / 2} 2^{-n f(k)} \leq 2 \cdot 2^{-n f(k) / 2}=2^{-\Omega(n f(k))}$.

Next, we give another reduction from random $k$-SAT to planted $k$-SAT. This theorem is different from Lemma 7, in that, given a planted $k$-SAT algorithm that works in a certain regime of $m$, it implies a random $k$-SAT algorithm for all values of $m$.

Reminder of Theorem 2, Suppose there is an algorithm A for planted $k$-SAT $P(n, k, m)$, for all $m \geq 2^{k} \ln 2(1-f(k) / 2) n$, which finds a solution in time $2^{n(1-f(k))}$ and with probability $1-2^{-n f(k)}$, where $1 / k<f(k)=o_{k}(1)$. Then for any $m^{\prime}$, given a random $k$-SAT instance sampled from $R^{+}\left(n, k, m^{\prime}\right)$, a satisfying assignment can be found in $2^{n(1-\Omega(f(k)))}$ time w.h.p.

Proof. Let $F$ be sampled from $R^{+}(n, k, m)$, and let $Z$ denote its number of solutions with $s$ its expected value. The expected number of solutions of $F^{\prime}$ sampled from $R\left(n, k, m^{\prime}\right)$ serves as a lower bound for $s$. Hence if $m^{\prime} \leq 2^{k} \ln 2(1-f(k) / 2) n \leq \alpha_{d} n$, then $s>2^{n f(k) / 2}$ and furthermore, as we have $f(k)>1 / k$, Lemma 3 implies that, $\lim _{n \rightarrow \infty} \operatorname{Pr}\left[Z<s / 2^{O\left(n k / 2^{k}\right)}\right]=$ 0 . So if we randomly sample $O\left(2^{n} 2^{O\left(n k / 2^{k}\right)} / s\right) \leq 2^{n(1-\Omega(f(k)))}$ assignments, one of them will satisfy $F$ w.h.p. Otherwise if $m^{\prime} \geq 2^{k} \ln 2(1-f(k) / 2) n$ then we can use the algorithm $A$ combined with Lemma 7 to solve it in required time.

Finally, we combine Algorithm 1 for planted $k$-SAT and the reduction in Theorem 2 to obtain an algorithm for finding solutions of random $k$-SAT (conditioned on satisfiability). This disproves Super-SETH for random $k$-SAT.

Reminder of Theorem 3. Given a random $k$-SAT instance $F$ sampled from $R^{+}(n, k, m)$ we can find a solution in $2^{n\left(1-\Omega\left(\frac{\log k}{k}\right)\right)}$ time w.h.p.

Proof. By Theorem 1 we have an algorithm for planted $k$-SAT running in $2^{n\left(1-\Omega\left(\frac{\log k}{k}\right)\right)}$ time with $1-2^{-\Omega\left(n\left(\frac{\log k}{k}\right)\right)}$ probability for all $m>\left(2^{k-1} \ln 2\right) n$. This algorithm satisfies the required conditions in Theorem 2 with $f(k)=\Omega(\log k / k)$ for large enough $k$. The implication in Theorem 2 proves the required statement.

Just as in the case of planted $k$-SAT, when $m<n\left(2^{k} \ln 2-k\right)$ we can find solutions of $R^{+}(n, k, m)$ w.h.p., by merely random sampling assignments. The correctness of random sampling follows from Lemma 3 . 


\section{Planted and Random $k$-SAT for large $m$}

In Sections 3 and 4 we gave algorithms for random $k$-SAT that work at the threshold and for all other values of the clause density. In this section, we work in the regime where the number of clauses $m$ is bounded away from the threshold, and give an improved running time analysis for this case. The proofs follow a similar structure to the proofs in Section 3 and 4. As mentioned before, polynomial-time algorithms finding solutions to random $k$-SAT instances currently require $m$ to be at least $\frac{4^{k}}{k} n$. To our knowledge, no improved algorithms were known for $2^{k} n<m<\frac{4^{k}}{k} n$ other than the worst case $k$-SAT algorithms.

Lemma 8. For $2^{k} n<m<2^{k+o(k)} n$, a planted $k$-SAT instance sampled from $P(n, k, m, \sigma)$ has $\Omega(n z)$ good variables with respect to $\sigma$, with probability $1-2^{-\Omega(n z)}$ where $z=(\ln (m / n)-$ $k \ln 2) / k$.

Proof. In this proof, by "good/bad variables" we mean "good/bad variables with respect to $\sigma$ " (see Section 3 to recall the definition of good/bad).

Let $F \in_{r} P(n, k, m, \sigma)$ and let $L$ be the last (when sorted by index) $n z / 2$ variables. Let $L_{g}, L_{b}$ be the good and bad variables respectively, with respect to $\sigma$, among $L$. Let $E$ denote the event that $\left|L_{g}\right| \leq n z / 500$.

We will prove a strong upper bound on the probability that $E$ occurs. For any $x_{i} \in L$, we have that $i \geq n(1-z / 2)$. If a clause $C$ is good with respect to $x_{i} \in L_{b}$, then we know that $C$ does not occur in $F$. Next, we will lower bound the probability of such a clause occurring with respect to a fixed variable $x_{i} \in L$. Recall that in planted $k$-SAT, each clause is drawn uniformly at random from the set of all clauses satisfying $\sigma$. We derive:

$$
\begin{aligned}
& \operatorname{Pr}\left[C \text { is good with respect to } x_{i} \in L\right] \\
& =\frac{\text { Number of clauses which will make } x_{i} \in L \text { good }}{\text { Total number of clauses which satisfy } \sigma} \\
& =\frac{\left(\begin{array}{l}
i-1 \\
k-1
\end{array}\right)}{\left(\begin{array}{l}
n \\
k
\end{array}\right)\left(2^{k}-1\right)} \\
& \geq \frac{1}{2}\left(\frac{i}{n}\right)^{k} \frac{k}{2^{k} n} \quad[\text { since } i \geq n(1-z / 2), z=o(1)] \\
& \geq \frac{1}{2}\left(1-\frac{z}{2}\right)^{k} \frac{k}{2^{k} n} \quad[\text { since } i \geq n(1-z / 2)] \\
& \geq \frac{1}{2}\left(e^{-z}\right)^{k} \frac{k}{2^{k} n} \quad\left[\text { since } z=o(1) \text { and } e^{-w} \leq 1-w / 2 \text { for small enough } w>0\right] \\
& \geq \frac{e^{-z k}}{2^{k+1} n}
\end{aligned}
$$

If the event $E$ is true, then $\left|L_{b}\right|=|L|-\left|L_{g}\right|>n z / 4$. Consider such a fixed set $L_{b}$. Under our sampling procedure, every time we sample a clause $C$, the probability that $C$ makes some variable $x_{i} \in L_{b}$ good is at least $\frac{n z}{4} \cdot \frac{e^{-z k}}{2^{k+1} n} \geq \frac{z e^{-z k}}{2^{k+3}}$, as the sets of clauses which make different variables good are disjoint sets. Now we upper bound the probability of $E$ occurring: 


$$
\begin{aligned}
\operatorname{Pr}[E] & \leq \sum_{i=1}^{n z / 500} \operatorname{Pr}[\text { Exactly } i \text { good variables among the last } n z / 2 \text { variables }] \\
& \leq \sum_{i=1}^{n z / 500}\left(\begin{array}{c}
n z / 2 \\
i
\end{array}\right)\left(1-\frac{z e^{-z k}}{2^{k+3}}\right)^{m} \\
& \leq n\left(\begin{array}{c}
n z / 2 \\
n z / 500
\end{array}\right)\left(1-\frac{z e^{-z k}}{2^{k+3}}\right)^{n e^{z k} 2^{k}} \quad\left[\text { since } m=e^{z k} 2^{k} n\right] \\
& \leq n\left(\begin{array}{c}
n z / 2 \\
n z / 500
\end{array}\right)\left(e^{-\frac{z e^{-z k}}{2^{k+3}}}\right)^{n e^{z k} 2^{k}} \quad\left[\text { since } 1-x \leq e^{-x} \text { for } x>0\right] \\
& \leq n\left(\begin{array}{c}
n z / 2 \\
n z / 500
\end{array}\right)\left(e^{-\frac{n z}{8}}\right) \\
& \leq 2^{-\delta n z},
\end{aligned}
$$

for appropriately small but constant $\delta>0$. This proves the lemma statement.

Theorem 5. Given a planted $k$-SAT instance $F$ sampled from $P(n, k, m)$ with $2^{k+o(k)} n>$ $m>2^{k} n$ define $z=(\ln (m / n)-k \ln 2) / k$ and $z^{\prime}=z+\ln k / k$, we can find a solution of $F$ in $2^{n\left(1-\Omega\left(z^{\prime}\right)\right)}$ time with at least $1-2^{-\Omega\left(n z^{\prime}\right)}$ probability (over the planted $k$-SAT distribution and the randomness of the algorithm).

Proof. By Lemma 8, we know that with probability at least $1-p$ for $p=2^{-\Omega(n z)}$, the number of good variables in $F$ (wrt the hidden planted solution $\sigma$ ) is at least $\gamma n z$ for some $\gamma>0$. For such instances, one run of the PPZ algorithm will output $\sigma$ with probability at least $2^{-n(1-\gamma z)}$. Repeating the PPZ algorithm for poly $(\mathrm{n}) 2^{n(1-\gamma z)}$ times implies a success probability of at least $1-p$ for $p^{\prime}=2^{-n}$. The overall error probability is at most $p+p^{\prime} \leq$ $2^{-\Omega(n z)}$.

Also by Theorem 1, there exists a random $k$-SAT algorithm running in $2^{n\left(1-\Omega\left(\frac{\log k}{k}\right)\right)}$ time with $1-2^{-\Omega\left(n\left(\frac{\log k}{k}\right)\right)}$ success probability. Together, these algorithms imply an algorithm running in $2^{n\left(1-\Omega\left(z^{\prime}\right)\right)}$ time with $1-2^{-\Omega\left(n z^{\prime}\right)}$ probability (over the planted $k$-SAT distribution and the randomness of the algorithm).

Theorem 6. Given a random $k$-SAT instance $F$ sampled from $R^{+}(n, k, m)$ with $2^{k+o(k)} n>$ $m>2^{k} n$ define $z=(\ln (m / n)-k \ln 2) / k$ and $z^{\prime}=z+\ln k / k$, we can find a solution of $F$ in $2^{n\left(1-\Omega\left(z^{\prime}\right)\right)}$ time with $1-2^{-\Omega\left(n z^{\prime}\right)}$ probability (over the random $k$-SAT distribution $R^{+}$and the randomness of the algorithm).

Proof. This follows directly from composing the algorithm in Theorem 5 and the reduction in Lemma 7 where we set $f(k)=z^{\prime}$.

As an example, the above theorem implies: For $F \in_{r} R^{+}(n, k, m)$ and $m=2^{k+\sqrt{k}} n$ we have a $2^{n(1-\Omega(1 / \sqrt{k}))}$ algorithm which works with $1-2^{-\Omega(n / \sqrt{k})}$ probability.

Next we will increase $m$ even further, and prove there are more good variables for the PPZ algorithm in this case. 
Lemma 9. Let $\varepsilon \in(0,1)$ and $t=\frac{2}{1-\varepsilon}>2$. Given a planted $k$-SAT instance $F$ sampled from $P(n, k, m, \sigma)$ with $m \geq t^{k} n, F$ has at least $\varepsilon n(1-2 / k)$ good variables with respect to the assignment $\sigma$, with probability $1-2^{-\Omega(\varepsilon n)}$.

Proof. The proof is similar to that of Lemma 8. As in that proof, by "good/bad variables" we mean "good/bad variables with respect to the assignment $\sigma$ ".

Let $F \in_{r} P(n, k, m, \sigma)$ and let $L$ be the last (when sorted by index) $\varepsilon n$ variables. Let $L_{g}, L_{b}$ be the good and bad variables respectively, with respect to $\sigma$, among $L$. Let $E$ be the event that $\left|L_{b}\right|>\gamma \varepsilon n$, where $\gamma=2 / k$. When $E$ is false we have $\left|L_{g}\right|>|L|-\left|L_{b}\right| \geq$ $\varepsilon n-\gamma \varepsilon n=\varepsilon n(1-2 / k)$ which is what we want to prove.

Analogously to previous cases, we want to give a strong upper bound on the probability that event $E$ occurs. For any $x_{i} \in L$, we have that, $i \geq n(1-\varepsilon)$. If clause $C$ is good with respect to $x_{i} \in L_{b}$, then we know $C$ does not occur in $F$. As before, our next step is to lower bound the probability of such a clause occurring with respect to a fixed variable $x_{i} \in L$. Recall that in planted $k$-SAT, each clause is drawn uniformly at random from the set of all clauses which satisfy $\sigma$. Therefore

$\operatorname{Pr}\left[C\right.$ is good with respect to $\left.x_{i} \in L\right]=\frac{\text { Number of clauses which will make } x_{i} \in L \text { good }}{\text { Total number of clauses which satisfy } \sigma}$

$$
\begin{aligned}
& =\frac{\left(\begin{array}{l}
i-1 \\
k-1
\end{array}\right)}{\left(\begin{array}{l}
n \\
k
\end{array}\right)\left(2^{k}-1\right)} \\
& \left.\geq \frac{1}{2}\left(\frac{i}{n}\right)^{k} \frac{k}{2^{k} n} \quad \text { since } i \geq n(1-\varepsilon)=\Omega(n)\right] \\
& \geq \frac{1}{2}(1-\varepsilon)^{k} \frac{k}{2^{k} n} \quad[\text { since } i \geq n(1-\varepsilon)] \\
& =\frac{k(1-\varepsilon)^{k}}{2^{k+1} n}
\end{aligned}
$$

If $E$ is true, then $\left|L_{b}\right|>\gamma \varepsilon n$. So the probability of sampling a clause such that there exists a variable $x_{i} \in L_{b}$ which is good with respect to the clause is at least $\frac{\gamma \varepsilon k(1-\varepsilon)^{k}}{2^{k+1}}$, as the sets of clauses which make different variables good are disjoint sets. Our upper bound on the event $E$ is then calculated as follows: 


$$
\begin{aligned}
\operatorname{Pr}[E] & \leq \sum_{i=1}^{\varepsilon n(1-\gamma)} \operatorname{Pr}[\text { Exactly } i \text { good variables among the last } \varepsilon n \text { variables }] \\
& \leq \sum_{i=1}^{\varepsilon n(1-\gamma)}\left(\begin{array}{c}
\varepsilon n \\
i
\end{array}\right)\left(1-\frac{\gamma \varepsilon k(1-\varepsilon)^{k}}{2^{k+1}}\right)^{m} \\
& \leq 2^{\varepsilon n}\left(1-\frac{\gamma \varepsilon k(1-\varepsilon)^{k}}{2^{k+1}}\right)^{t^{k} n} \quad\left[\text { since } m>t^{k} n\right] \\
& \leq 2^{\varepsilon n}\left(1-\frac{\varepsilon(1-\varepsilon)^{k}}{2^{k}}\right)^{t^{k} n}[\gamma=2 / k] \\
& \leq 2^{\varepsilon n}\left(1-\frac{\varepsilon 2^{k}}{t^{k} 2^{k}}\right)^{t^{k} n}[\text { since } 1-\varepsilon=2 / t] \\
& \leq 2^{\varepsilon n}\left(1-\frac{\varepsilon}{t^{k}}\right)^{t^{k} n} \\
& \leq 2^{\varepsilon n} e^{-\varepsilon n}\left[\text { since } 1-x \leq e^{-x} \text { for } x>0\right] \\
& \leq 2^{-\delta \varepsilon n},
\end{aligned}
$$

for appropriately small but constant $\delta>0$. This proves the lemma statement.

Theorem 7. Let $\varepsilon \in(0,1)$ and $t=\frac{2}{1-\varepsilon}>2$. Given a planted $k$-SAT instance $F$ sampled from $P(n, k, m)$ with $m \geq t^{k} n$, we can find a solution of $F$ in $2^{n(1-\varepsilon(1-2 / k))}$ poly(n) time with $1-2^{-\Omega(\varepsilon n)}$ probability (over the planted $k$-SAT distribution and the randomness of the algorithm).

Proof. By Lemma 9, there is probability at least $1-p$ for $p=2^{-\Omega(\varepsilon n)}$ that the number of good variables in $F$ is at least $\varepsilon n(1-2 / k)$ with respect to the hidden planted solution $\sigma$. For such instances, one run of the PPZ algorithm outputs $\sigma$ with probability at least $2^{-n(1-\varepsilon(1-2 / k))}$. Repeating PPZ for poly $(\mathrm{n}) 2^{n(1-\varepsilon(1-2 / k))}$ times implies success probability at least $1-p^{\prime}$ for $p^{\prime}=2^{-n}$. The overall error probability is at most $p+p^{\prime} \leq 2^{-\Omega(n(1-2 / t))}$.

In order to use Theorem 7 to obtain algorithms for $R^{+}$, we need a more refined version of Lemma 7 .

Lemma 10. Suppose there is an algorithm A for planted $k$-SAT $P(n, k, m)$ for some $m \geq$ $\alpha_{\text {sat }} n$ which finds a solution in time $2^{n(1-f(k))}$ and with probability $\geq 1-p$. Then, given a random $k$-SAT instance $F$ sampled from $R^{+}(n, k, m)$, we can find a solution to $F$ in $2^{n(1-f(k))}$ time with at least $1-p \cdot 2^{O\left(n / 2^{k}\right)}$ probability.

Proof. Let $F$ be sampled from $R^{+}(n, k, m)$, let $Z$ denote the number of solutions, and let $s$ be its expected value. Since $m \geq \alpha_{\text {sat }} n$, Lemma 5 implies $s \leq 2^{O\left(n / 2^{k}\right)}$.

Suppose we simply run Algorithm $A$. If Algorithm $A$ gives a solution, it is correct, hence our only source of error is when the formula is satisfiable but algorithm $A$ does not return a 
solution. We can upper bound the probability of $A$ making an error in this way as follows:

$$
\begin{aligned}
& \underset{F \in R^{+}(n, k, m), A}{\operatorname{Pr}}[\text { A does not return a solution] } \\
& \leq \sum_{\sigma \in\{0,1\}^{n}} \operatorname{Pr}_{F \in R^{+}(n, k, m), A}[\sigma \text { satisfies } \mathrm{F} \text { but } A \text { does not return a solution }] \\
& \leq \sum_{\sigma \in\{0,1\}^{n}} \operatorname{Pr}_{F \in R^{+}(n, k, m), A}[A \text { does not return a solution } \mid \sigma \text { satisfies } \mathrm{F}] \operatorname{Pr}_{F \in R^{+}(n, k, m)}[\sigma \text { satisfies } \mathrm{F}] \\
& \leq \sum_{\sigma \in\{0,1\}^{n}} \operatorname{Pr}_{F \in P(n, k, m, \sigma), A}[A \text { does not return a solution }] \underset{F \in R^{+}(n, k, m)}{\operatorname{Pr}}[\sigma \text { satisfies F }],
\end{aligned}
$$

where the last inequality used the fact that (by definition) $R^{+}(n, k, m)$ conditioned on having $\sigma$ as a solution is exactly $P(n, k, m, \sigma)$.

Note that $\operatorname{Pr}_{F \in R^{+}(n, k, m)}[\sigma$ satisfies $\mathrm{F}]=s / 2^{n}$ and $P(n, k, m)$ is just $P(n, k, m, \sigma)$ where $\sigma$ is sampled uniformly from $\{0,1\}^{n}$. Hence the above expression simplifies to

$=\frac{s}{2^{n}}\left(2^{n} \operatorname{Pr}_{F \in P(n, k, m), A}[A\right.$ does not return a solution $\left.]\right)=s \operatorname{Pr}_{F \in P(n, k, m), A}[A$ does not return a solution $]$.

Since $s \leq 2^{O\left(n / 2^{k}\right)}$, the error probability is at most $p \cdot 2^{O\left(n / 2^{k}\right)}$.

Theorem 8. Let $\varepsilon \in(0,1)$ and $t=\frac{2}{1-\varepsilon}>2$. For a large enough $k$, given a random $k$-SAT instance $F$ sampled from $R^{+}(n, k, m)$ with $m \geq t^{k} n$, we can find a solution of $F$ in $2^{n(1-\varepsilon(1-2 / k))}$ poly $(n)$ time with $1-2^{-\Omega(\varepsilon n)}$ probability (over the planted $k$-SAT distribution and the randomness of the algorithm).

Proof. The algorithm in Theorem 7 and the reduction in Lemma 10 imply that we can find a solution of $F$ in $2^{n(1-\varepsilon(1-2 / k))}$ poly(n) time with $1-2^{O\left(n / 2^{k}\right)} 2^{-\Omega(\varepsilon n)}=1-2^{-\Omega(\varepsilon n)}$ probability, for a large enough $k$.

\section{6. k-SAT and Unique k-SAT}

In this section we give a randomized reduction from $k$-SAT to Unique $k$-SAT which implies their equivalence for Super Strong ETH:

Reminder of Theorem 4. If Unique $k$-SAT is solvable in $2^{(1-f(k) / k) n}$ time for some unbounded $f(k)$, then $k-S A T$ is solvable in $2^{(1-f(k) / k+O((\log f(k)) / k)) n}$ time.

We start with a slight modification of the Valiant-Vazirani lemma.

Lemma 11 (Weighted-Valiant-Vazirani). Let $S \subseteq\{0,1\}^{k}=R$ be a set of assignments on variables $x_{1}, x_{2}, \ldots x_{k}$, with $2^{j-1} \leq|S|<2^{j}$. Suppose that for each $s \in S$ there exists a weight $w_{s} \in \mathbb{Z}^{+}$, and let $\bar{w}$ denote the average weight over all $s \in S$. There is a randomized polytime algorithm Weighted-Valiant-Vazirani that on input $(R, j)$ outputs a matrix $A \in \mathbb{F}_{2}^{j \times n}$ and a vector $b \in \mathbb{F}_{2}^{j}$ such that

$$
\underset{A, b}{\operatorname{Pr}}\left[|\{x \mid A x=b \wedge x \in S\}|=1, w_{s} \leq 2 \bar{w}\right]>\frac{1}{16} .
$$


If the condition in the probability expression is satisfied, we say Weighted-Valiant-Vazirani on $(R, j)$ has succeeded.

Proof. The original Valiant-Vazirani Lemma (Valiant \& Vazirani, 1986) gives a randomized polytime algorithm to generate $A, b$ such that for all $s \in S, \operatorname{Pr}_{A, b}[\{s\}=\{x \mid A x=b \wedge x \in$ $S\}]>\frac{1}{8|S|}$. Moreover, by Markov's inequality, we have $\operatorname{Pr}_{s \in S}\left[w_{s} \leq 2 \bar{w}\right] \geq 1 / 2$. Hence the set of $s \in S$ with $w_{s} \leq 2 \bar{w}$ has size at least $|S| / 2$. This implies $\operatorname{Pr}_{A, b}[\exists s,\{s\}=\{x \mid A x=$ $\left.b \wedge x \in S\}, w_{s} \leq 2 \bar{w}\right]>\left(\frac{1}{8|S|}\right)\left(\frac{|S|}{2}\right)=\frac{1}{16}$.

Proof of Theorem 4. Let $A$ be an algorithm for Unique $k$-SAT which runs in time $2^{(1-f(k) / k) n}$.

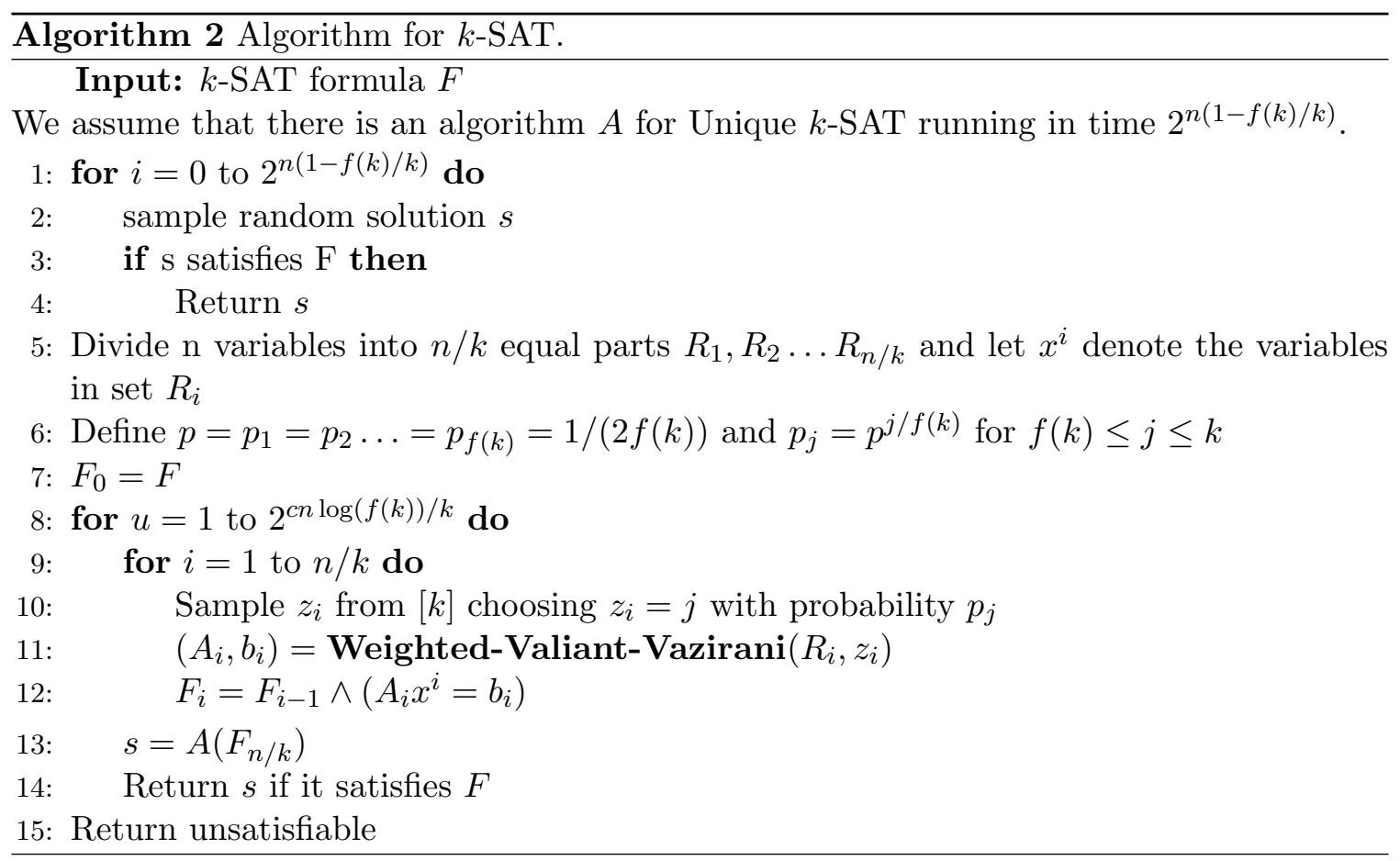

Let $S$ be the set of SAT assignments to $F$. Suppose $|S| \geq 2^{n f(k) / k} n$. Then the probability that the random search in lines 1 to 4 never finds a solution is

$$
\left(1-n 2^{n f(k) / k} / 2^{n}\right)^{2^{n(1-f(k) / k)}} \leq e^{-n}
$$

Thus if $|S| \geq 2^{n f(k) / k} n$ then the algorithm finds a solution w.h.p. From now on, we assume $|S|<2^{n f(k) / k} n$.

In line 6, we define a sequence of probabilities $p_{1}, p_{2}, \ldots, p_{k}$. Note that

$$
\begin{aligned}
\sum_{i=1}^{k} p_{i} & =\sum_{i=1}^{f(k)} p_{i}+\sum_{i=f(k)+1}^{k} p_{i} \leq 1 / 2+1 /(2 f(k)) \sum_{j=1}^{\infty}(1 / 2 f(k))^{j / f(k)} \\
& \leq \frac{1}{2}+\frac{1}{f(k)\left(1-(1 / 2 f(k))^{1 / f(k)}\right)} \leq 1
\end{aligned}
$$


as $f(k)$ is unbounded, and $\lim _{x \rightarrow \infty} x\left(1-(1 / 2 x)^{1 / x}\right)=\infty$.

We will now analyze the $i^{\text {th }}$ run of the loop from line 9 to line 14 . Let $S_{0}=S$, and let $S_{i}$ be the set of solutions to the formula $F_{i}$ defined in line 12 .

Let $E_{i}$ be the event that:

1. $2^{z_{i}-1} \leq\left|\left\{s_{\mid R_{i}} \mid s \in S_{i-1}\right\}\right|<2^{z_{i}}$. [As defined in line 10$]$

2. for all $s \in S_{i}$, the restriction on $R_{i}$ is the same, i.e., $\left|\left\{s_{\mid R_{i}} \mid s \in S_{i}\right\}\right|=1$.

3. $\left|S_{i-1}\right| /\left|S_{i}\right| \geq 2^{z_{i}-2},\left|S_{i}\right| \neq 0$.

Let $y_{i}$ satisfy $2^{y_{i}-1} \leq\left|\left\{s_{\mid R_{i}} \mid s \in S_{i-1}\right\}\right|<2^{y_{i}}$. In Line 11 we apply WeightedValiant-Vazirani to $\left(R_{i}, z_{i}\right)$ with the set of assignments being $\left\{s_{\mid R_{i}} \mid s \in S_{i-1}\right\}$ where an assignment $v$ has weight $w_{v}=\left|\left\{s \mid v=s_{\mid R_{i}} \wedge s \in S_{i-1}\right\}\right|$ i.e. $w_{v}=$ number of solutions of $F_{i-1}$ with $v$ as the restriction on $R_{i}$. For Weighted-Valiant-Vazirani to apply, we require that $z_{i}$ indeed represents an estimate of number of possible assignments to variables of $R_{i}$ in a satisfying assignment i.e. $2^{z_{i}-1} \leq\left|\left\{s_{\mid R_{i}} \mid s \in S_{i-1}\right\}\right|<2^{z_{i}}$ which is exactly the condition 1 in $E_{i}$. Stated in other words, we require $z_{i}=y_{i}$. If the call to WeightedValiant-Vazirani succeeds, then we have that only a unique assignment to $R_{i}$ remains, i.e, $\left|\left\{s_{\mid R_{i}} \mid s \in S_{i}\right\}\right|=1$ which is the condition 2 of $E_{i}$. Denote this unique assignment to $R_{i}$ variables by $u$. Lemma 11 also states that if Weighted-Valiant-Vazirani succeeds then

$$
\left|S_{i}\right|=w_{u} \leq 2 \bar{w}=2 \cdot \frac{\sum_{s \in S_{i-1}, v=s_{\mid R_{i}}} w_{v}}{\left|\left\{s_{\mid R_{i}} \mid s \in S_{i-1}\right\}\right|} \leq 2 \cdot \frac{\left|S_{i-1}\right|}{2^{y_{i}-1}}=\frac{\left|S_{i-1}\right|}{2^{y_{i}-2}}
$$

Hence condition 3 is also implied if $z_{i}=y_{i}$ and Weighted-Valiant-Vazirani succeeds.

Hence for $E_{i}$ to be true we need that the sample $z_{i}$ is equal to $y_{i}$, and WeightedValiant-Vazirani on $\left(R_{i}, z_{i}\right)$ succeeds.

Let $E=\bigcap_{i} E_{i}$. If event $E$ occurs, then the restrictions of all solutions on each $R_{i}$ 's are the same, and there is a solution as $\left|S_{n / k}\right| \neq 0$, hence there is a unique satisfying assignment. We wish to lower bound the probability of $E$ occurring.

$$
\begin{aligned}
\operatorname{Pr}[E] & =\prod_{i} \operatorname{Pr}\left[E_{i} \mid \bigwedge_{j<i} E_{j}\right] \\
& \geq \prod_{i} \operatorname{Pr}\left[z_{i}=y_{i} \mid \bigwedge_{j<i} E_{j}\right] \cdot \prod_{i} \operatorname{Pr}\left[\mathbf{W V V}\left(R_{i}, z_{i}\right) \mid \forall j<i, E_{j}\right] \\
& \geq \prod_{i} p_{y_{i}} \prod_{i}\left(\frac{1}{16}\right) \quad[\text { By Lemma 11] } \\
& \geq 16^{-n / k} \prod_{i} p_{y_{i}}
\end{aligned}
$$

When $E$ holds, $|S|=\left|S_{0}\right|=\prod_{i}\left|S_{i-1}\right| /\left|S_{i}\right|$, as $\left|S_{n / k}\right|=1$, Furthermore $\prod_{i}\left|S_{i-1}\right| /\left|S_{i}\right| \geq$ $\prod_{i} 2^{y_{i}-2}$, by condition 3 . Since $|S| \leq 2^{n f(k) / k} n$, we have $\prod_{i} 2^{y_{i}-2} \leq 2^{n f(k) / k} n$. Taking 
logarithms, $\sum_{i} y_{i} \leq O(n / k)+n f(k) / k \leq O(n f(k) / k)$. Therefore

$$
\begin{aligned}
\operatorname{Pr}[E] & \geq 16^{-n / k} \prod_{i} p_{y_{i}} \quad \text { Restating equation (1)] } \\
& \geq 16^{-n / k} \prod_{y_{i} \leq f(k)} p_{y_{i}} \prod_{y_{i}>f(k)} p_{y_{i}} \\
& \geq 16^{-n / k} \cdot(1 / 2 f(k))^{n / k} \cdot \prod_{y_{i}>f(k)}(1 / 2 f(k))^{\left(y_{i} / f(k)\right)} \\
& \geq 16^{-n / k} \cdot(1 / 2 f(k))^{n / k} \cdot(1 / 2 f(k))^{\sum_{y_{i}>f(k)}\left(y_{i} / f(k)\right)} \\
& \geq 16^{-n / k} \cdot(1 / 2 f(k))^{n / k} \cdot(1 / 2 f(k))^{O(n / k)} \\
& \geq 16^{-n / k} \cdot 2^{-O(n \log f(k) / k)} \geq 2^{-O(n \log f(k) / k)} .
\end{aligned}
$$

As mentioned earlier, if $E$ occurs, then there is a unique SAT assignment and it is found by our Unique $k$-SAT algorithm $A$. The probability $E$ does not happen over all $2^{c n(\log f(k)) / k}$ runs of the loop on line 8 is at most $\left.1-2^{-O(n(\log f(k)) / k)}\right)^{2^{c n(\log f(k)) / k}} \ll 2^{-n}$, for sufficiently large $c$. The total running time is $2^{n(1-f(k) / k)}+2^{c n(\log f(k)) / k} \cdot 2^{(1-f(k) / k) n} \leq$ $2^{(1-f(k) / k+O((\log f(k)) / k)) n}$.

Theorem 4 immediately implies an "ultra fine-grained" equivalence between $k$-SAT and Unique- $k$-SAT:

Corollary 2. Unique $k$-SAT is in $2^{\left(1-\omega_{k}(1 / k)\right) n}$ time $\Leftrightarrow k$-SAT is in $2^{\left(1-\omega_{k}(1 / k)\right) n}$ time.

\section{Conclusion}

We have shown two significant results regarding the hypothesis that $k$-SAT cannot be solved much faster than $2^{n(1-\Theta(1 / k))}$ time (the Super Strong ETH). First, we showed that for random $k$-SAT instances, simple unit-clause propagation combined with random variable choice (and random restarts with no backtracking) can be used to solve SAT faster than what is known in the worst case, refuting the Super Strong ETH for random instances. Our algorithm runs in $2^{n(1-\Theta(\log k) / k)}$ time. We also showed even slightly faster algorithms for solving Unique $k$-SAT would imply similar algorithms for general $k$-SAT. Thus, to refute the Super Strong ETH, we may assume without loss of generality that the $k$-SAT instances given to our algorithms have at most one satisfying assignment.

This paper is an extended version of the conference paper (Vyas \& Williams, 2019) which appeared in SAT'19. Very recently, another algorithm for random $k$-SAT has been presented (based on local search, instead of unit-clause propagation) that achieves a faster running time of $2^{n\left(1-\Omega\left(\log ^{2} k\right) / k\right)}$ (Lincoln \& Yedidia, 2020).

\section{Acknowledgment}

Authors were supported by NSF CCF-1741615 and NSF CCF-1909429. 


\section{References}

Achlioptas, D., \& Coja-Oghlan, A. (2008). Algorithmic barriers from phase transitions. In Foundations of Computer Science, 2008. FOCS'08. IEEE 49th Annual IEEE Symposium on, pp. 793-802. IEEE.

Ben-Sasson, E., Bilu, Y., \& Gutfreund, D. (2002). Finding a randomly planted assignment in a random $3 \mathrm{cnf}$.

Brakensiek, J., \& Guruswami, V. (2019). Bridging between 0/1 and linear programming via random walks. In Charikar, M., \& Cohen, E. (Eds.), Proceedings of the 51st Annual ACM SIGACT Symposium on Theory of Computing, STOC 2019, Phoenix, AZ, USA, June 23-26, 2019, pp. 568-577. ACM.

Calabro, C., Impagliazzo, R., Kabanets, V., \& Paturi, R. (2008). The complexity of unique k-sat: An isolation lemma for k-cnfs. Journal of Computer and System Sciences, 74 (3), 386-393.

Calabro, C., Impagliazzo, R., \& Paturi, R. (2009). The complexity of satisfiability of small depth circuits. In Parameterized and Exact Computation, 4th International Workshop, IWPEC 2009, Copenhagen, Denmark, September 10-11, 2009, Revised Selected Papers, pp. 75-85.

Chan, T. M., \& Williams, R. (2016). Deterministic apsp, orthogonal vectors, and more: Quickly derandomizing razborov-smolensky. In Proceedings of the Twenty-Seventh Annual ACM-SIAM Symposium on Discrete Algorithms, SODA 2016, Arlington, VA, USA, January 10-12, 2016, pp. 1246-1255.

Chao, M.-T., \& Franco, J. (1990). Probabilistic analysis of a generalization of the unit-clause literal selection heuristics for the $\mathrm{k}$ satisfiability problem. Information Sciences: an International Journal, 51(3), 289-314.

Coja-Oghlan, A. (2010). A better algorithm for random k-sat. SIAM Journal on Computing, $39(7), 2823-2864$.

Coja-Oghlan, A., Krivelevich, M., \& Vilenchik, D. (2007). Why almost all satisfiable k-cnf formulas are easy..

Cook, S. A., \& Mitchell, D. G. (1996). Finding hard instances of the satisfiability problem: A survey. In Satisfiability Problem: Theory and Applications, Proceedings of a DIMACS Workshop, Piscataway, New Jersey, USA, March 11-13, 1996, pp. 1-18.

Ding, J., Sly, A., \& Sun, N. (2015). Proof of the satisfiability conjecture for large k. In Proceedings of the Forty-Seventh Annual ACM on Symposium on Theory of Computing, STOC 2015, Portland, OR, USA, June 14-17, 2015, pp. 59-68.

Feige, U. (2002). Relations between average case complexity and approximation complexity. In Proceedings on 34th Annual ACM Symposium on Theory of Computing, May 19-21, 2002, Montréal, Québec, Canada, pp. 534-543.

Gomes, C. P., Kautz, H. A., Sabharwal, A., \& Selman, B. (2008). Satisfiability solvers. In Handbook of Knowledge Representation, pp. 89-134.

Hertli, T. (2014). 3-sat faster and simpler - unique-sat bounds for PPSZ hold in general. SIAM J. Comput., 43(2), 718-729. 
Impagliazzo, R., \& Paturi, R. (2001). On the complexity of k-sat. J. Comput. Syst. Sci., 62 (2), 367-375.

Krivelevich, M., \& Vilenchik, D. (2006). Solving random satisfiable 3cnf formulas in expected polynomial time. In Proceedings of the Seventeenth Annual ACM-SIAM Symposium on Discrete Algorithms, SODA 2006, Miami, Florida, USA, January 22-26, 2006, pp. 454-463.

Lincoln, A., \& Yedidia, A. (2020). Faster random k-cnf satisfiability. In Czumaj, A., Dawar, A., \& Merelli, E. (Eds.), 47th International Colloquium on Automata, Languages, and Programming, ICALP 2020, July 8-11, 2020, Saarbrücken, Germany (Virtual Conference), Vol. 168 of LIPIcs, pp. 78:1-78:12. Schloss Dagstuhl - Leibniz-Zentrum für Informatik.

Paturi, R., Pudlák, P., Saks, M. E., \& Zane, F. (2005). An improved exponential-time algorithm for $k$-sat. J. $A C M$, 52(3), 337-364.

Paturi, R., Pudlák, P., \& Zane, F. (1999). Satisfiability coding lemma. Chicago J. Theor. Comput. Sci., 1999.

Pudlák, P., Scheder, D., \& Talebanfard, N. (2017). Tighter hard instances for PPSZ. In 44th International Colloquium on Automata, Languages, and Programming, ICALP 2017, July 10-14, 2017, Warsaw, Poland, pp. 85:1-85:13.

Scheder, D., \& Talebanfard, N. (2020). Super strong ETH is true for PPSZ with small resolution width. In Saraf, S. (Ed.), 35th Computational Complexity Conference, CCC 2020, July 28-31, 2020, Saarbrücken, Germany (Virtual Conference), Vol. 169 of LIPIcs, pp. 3:1-3:12. Schloss Dagstuhl - Leibniz-Zentrum für Informatik.

Schöning, U. (1999). A probabilistic algorithm for k-sat and constraint satisfaction problems. In 40th Annual Symposium on Foundations of Computer Science, FOCS '99, 17-18 October, 1999, New York, NY, USA, pp. 410-414.

Selman, B., Mitchell, D. G., \& Levesque, H. J. (1996). Generating hard satisfiability problems. Artificial intelligence, 81(1-2), 17-29.

Traxler, P. (2008). The time complexity of constraint satisfaction. In Parameterized and Exact Computation, Third International Workshop, IWPEC 2008, Victoria, Canada, May 14-16, 2008. Proceedings, pp. 190-201.

Valiant, L. G., \& Vazirani, V. V. (1986). NP is as easy as detecting unique solutions. Theor. Comput. Sci., 47(3), 85-93.

Vilenchik, D. (2019) personal communication.

Vyas, N., \& Williams, R. R. (2019). On super strong ETH. In Theory and Applications of Satisfiability Testing - SAT 2019 - 22nd International Conference, SAT 2019, Lisbon, Portugal, July 9-12, 2019, Proceedings, pp. 406-423.

Williams, R. (2015). Circuit analysis algorithms. Talk at Simons Institute for Theory of Computing, available at https://youtu.be/adJvi7tL-qM?t=925. 